\title{
Role of the anterior temporal lobes in semantic representations: Paradoxical results of a cTBS study
}

\author{
Sonia Bonnì ${ }^{\mathrm{a}, *}$, Giacomo Koch ${ }^{\mathrm{a}, \mathrm{b}}$, Carlo Miniussi ${ }^{\mathrm{c}, \mathrm{d}}$, Mario Stampanoni Bassi ${ }^{\mathrm{e}}$, \\ Carlo Caltagirone ${ }^{\mathrm{a}, \mathrm{b}}$, Guido Gainotti ${ }^{\mathrm{a}, \mathrm{f}}$ \\ a Non-invasive Brain Stimulation Unit, Santa Lucia Foundation IRCCS, Rome, Italy \\ b Department of Neuroscience, "Tor Vergata" University, Rome, Italy \\ ' Cognitive Neuroscience Section, IRCCS Centro San Giovanni di Dio Fatebenefratelli, Brescia, Italy \\ d Neuroscience Section, Department of Clinical and Experimental Sciences, University of Brescia, Italy \\ e Department of Neurology, "Tor Vergata" University, Rome, Italy \\ ${ }^{\mathrm{f}}$ Center for Neuropsychological Research, Institute of Neurology, Catholic University of Rome, Rome, Italy
}

\section{A R T I C L E I N F O}

\section{Article history:}

Received 30 June 2014

Received in revised form

31 October 2014

Accepted 3 November 2014

Keywords:

'Semantic Hub' model

Continuous theta burst stimulation

Virtual lesion account

Right and left anterior temporal lobes

Lexical-semantic and pictorial

representations.

\begin{abstract}
A B S T R A C T
According to the 'Semantic Hub' model, which was developed from data gathered in the moderate to advanced stages of semantic dementia (SD), a unitary amodal mechanism, located in the anterior parts of both temporal lobes (ATLs), should support the interactive activation of semantic representations in all modalities and for all semantic categories. This model has been challenged by clinical findings, which show that in the early stages of SD, when important asymmetries can be observed at the level of the right and left ATLs, the semantic impairment can be modality-specific, mainly affecting lexical-semantic knowledge when the left temporal lobe is more atrophic and pictorial representations when atrophy prevails on the right side. On the other hand, findings of experiments conducted in normal subjects with repetitive transcranial magnetic stimulations (rTMS), support the unitary model. In the most compelling of these studies, rTMS was used to investigate the role of right and left ATLs directly, by comparing semantic processing of the same concepts, presented as written words or pictures. The efficiency of semantic processing for words and pictures was reduced to the same degree by rTMS applied to the left and right ATLs. However, to consider more in depth some methodological inconsistencies of these studies and with the aim of discussing the effects of rTMS on high-level cognitive functions, we decided to repeat that experimental paradigm, using the continuous theta burst stimulation (cTBS) protocol over the right ATL, left ATL and vertex (as control site). A significant interaction was found between side of cTBS application and type of stimulus, but, contrary to our predictions, we observed significantly faster (rather than slower) responses to pictures after application of cTBS to the right ATL and no difference between responses to written words after application of CTBS to the left ATL in comparison with the vertex. These unexpected results are discussed with respect to the nature of the semantic representations supported by the right and left ATLs and to re-appraisal of the 'virtual lesion' account to explain results obtained with rTMS experiments on high-level cognitive functions.
\end{abstract}

C 2014 Published by Elsevier Ltd.

\section{Introduction}

Patterson et al. (2007) and Lambon-Ralph and Patterson (2008) proposed the existence of a mechanism (i.e., the 'Semantic Hub') in the anterior parts of both temporal lobes (ATLs), which supports the interactive activation of semantic representations in all modalities and for all semantic categories. This model was suggested

\footnotetext{
* Correspondence to: Non-invasive Brain Stimulation Unit, Laboratorio di Neurologia Clinica e Comportamentale, IRCCS Fondazione S. Lucia via Ardeatina 306, 00179 Rome, Italy.

E-mail address: s.bonni@hsantalucia.it (S. Bonnì).
}

by the fact that the bilateral degeneration of the inferior and lateral parts of the ATLs, usually observed in the moderate to advanced stages of semantic dementia (SD), leads to a selective, cross-modal and widespread semantic impairment. This impairment can be observed in a context of intact day-to-day memory, perceptual and spatial abilities and (in the language domain) syntactic and phonological processing. According to Patterson et al. (2007) and Lambon-Ralph and Patterson (2008), the 'Semantic Hub' should contain abstract and amodal semantic representations, because 'the range of concepts over which a component of knowledge should be generalized requires (amodal) representations that abstract away from surface similarities' 
(Lambon-Ralph and Patterson, 2008). However, at variance with this assumption, some data suggest that in the early stages of SD, when important asymmetries can be observed at the level of the right and left ATLs, the semantic impairment can be modalityspecific. In these cases, it mainly affects lexical-semantic knowledge when the left temporal lobe is more atrophic and pictorial representations when atrophy prevails on the right side. Indeed, it is worth noting that the first case of SD reported by the Cambridge group (Hodges et al., 1992) concerned a patient (FM), who showed a purely verbal disorder, namely a severe anomia and a mild lexical comprehension impairment in the absence of non-verbal semantic disorders, which was associated with infero-lateral atrophy of the left temporal lobe. Subsequently, this patient revealed a pattern characterized by a progressive, selective defect in word finding, without relevant verbal or non-verbal semantic disorders, which was labelled 'progressive pure anomia' by Graham et al. (1995). Some years later, Lambon Ralph et al. (2001) gathered longitudinal data in 16 patients with SD, to determine whether the enduring discrepancy between severity of anomia and semantic disorders shown by patient FM was exceptional or could be observed in other SD patients. Results of this study confirmed that two different patterns of disease progression can be observed in SD patients: the first is characterized by a parallel decline in naming and semantic-lexical comprehension, whereas the second is characterized by a progressive anomia, without a commensurate decline in semantic-lexical comprehension. Furthermore, several authors (e.g., Antonucci et al., 2008; Mesulam et al., 2013) confirmed that in patients with inferior temporal lobe lesions the degree of disparity between severity of anomia and the semanticlexical comprehension impairment is associated with that of the asymmetry in the laterality of lesions. The reverse pattern of impairment, namely an association between a prevalent disruption of non-verbal representations and a greater atrophy of the right ATL has also been described by several authors. Thus, Gorno-Tempini et al. (2004) reported a detailed assessment of a case (JT) of right temporal lobe variant of fronto temporal dementia. In addition to early and prominent behavioural changes, JT showed a disproportionate impairment on the pictures (in comparison with the words) version of the 'Pyramids and Palm Trees' (PPT) test (Howard and Patterson, 1992), which measures semantic association and involves matching either words or pictures based on semantic relatedness. Results consistent with the assumption that the semantic impairment mainly affects lexical-semantic knowledge when the left temporal lobe is more atrophic and pictorial representations when atrophy prevails on the right side have also been reported in group studies by Snowden et al. (2004, 2012). These authors administered tests of famous faces and names and the verbal and pictorial versions of the PPT test to SD patients, whose ATL atrophy was more severe on the left or on the right side. They found that subjects with a predominance of left ATL atrophy (a) identified famous people better from faces than from names, and (b) obtained better results on the pictorial than on the verbal version of the PPT test; by contrast, patients with more severe right ATL atrophy showed the opposite pattern of results. In addition to studies that assessed the neural correlates of verbal and nonverbal semantic processing deficits in patients with right and left ATL atrophy, the findings of some functional neuroimaging investigations (reviewed by Gainotti, 2011, 2012) also provided support for the hypothesis which assumes that conceptual representations can be stored in a partly different (mainly verbal and mainly non-verbal) format in the left and right ATL. The mechanisms through which conceptual representations could be preferentially stored in a verbal format in the left ATL and in a sensori-motor (pictorial) format in the right ATL are only hypothetical, It is possible, however, that each ATL constitutes the top of a series of hierarchically organized 'convergence zones'
(Damasio, 1989, 1990), where the various attributes of a concept are linked. In this case, the prevalence of non-verbal representations at the level of the right ATL might reflect the greater role of sensory-motor forms of knowledge in the right hemisphere, just as the prevalence of verbally coded representations in the left ATL might reflect the leading role of language in the left hemisphere. In contrast with the above mentioned clinical and experimental results, a series of experiments conducted in normal subjects by Pobric et al. (2007, 2010a, 2010b, 2009) and Lambon-Ralph et al. (2009) using repetitive transcranial magnetic stimulations (rTMS) support the hypothesis which assumes that the same abstract and amodal representations are equally subsumed by both the left and the right ATLs. In the most compelling of these studies, Pobric et al. (2010a) used rTMS to investigate the role of right and left ATLs directly, by comparing semantic processing for the same concepts, presented as written words or pictures, with the processing of purely perceptual (scrambled) stimuli. The efficiency of semantic processing for words and pictures was reduced to the same degree by rTMS applied to the left and the right ATLs, but the purely perceptual task was unaffected. Even if the authors considered their results as contrasting with the idea of two differentially specialised semantic hubs in the left and right ATL, they did not conclude that there are no subtle, graded differences in processing across the two hemispheres, but only that both hemispheres are involved in both word and picture versions of the task. The authors also commented that it was possible that stimulating the left ATL could have induced inhibition or alternatively compensatory excitation in the contralateral side (right ATL), but that, in any case, by stimulating both sites they had confirmed that both the left and the right ATL are part of the functional network which supports semantic cognition. Turning to the methodological aspects of the experiment, it is worth noting that a middle occipital stimulation site (occipital pole) was also employed as a site of control for possible non-specific visual effects. The occipital site $(\mathrm{Oz})$ had already been used as a control site in previous TMS studies on semantics by Knecht et al. (2002) and by Fuggetta et al. (2009) but, in Pobric et al.'s (2010a) study, responses were faster after rTMS application over the occipital pole, in contrast with the delayed processing of pictures and words caused by stimulation of the right and left ATLs. Therefore we decided not to use the occipital pole to avoid any possible indirect influence on the task. The observation of faster responses after rTMS in a control site is not surprising, because in a pilot study the authors had found that rTMS was highly alerting for participants irrespective of site of stimulation and might, therefore, produce a non-specific speeding of reaction times (RT) on all tasks. What is surprising, however, is the observation that a stimulation, which in the past was defined as a 'virtual lesion' of the occipital pole, produced faster responses to all sorts of visual stimuli. Thus, it seems logical to expect dissociations between the impairment of all kinds of visual stimuli after rTMS application over the primary visual areas and selective impairment of written words and pictures after stimulation of the right and left ATLs. It is less easy to explain why stimulation of the primary visual areas speeds up the processing of all kinds of visual stimuli, whereas similar stimulation of the right and left ATLs delays the processing of written words and pictures. Although the 'virtual lesion' account of results obtained with rTMS experiments has been useful to interpret the behavioural effects of non invasive brain stimulation, several TMS studies have also shown improved cognitive performance (for a review see Vallar and Bolognini, 2011) and have suggested new theoretical frameworks to better understand the TMS effects on cognitive tasks (Miniussi et al., 2010; Miniussi et al., 2013).

In light of these controversial issues, we investigated the specific involvement of the ATLs in semantic representation in a sample of healthy subjects, by using continuous theta burst 
stimulation (cTBS). We employed a modified version of Pobric et al.'s (2010a) experimental procedure, which consisted of using the cTBS, known for its 'inhibitory' long-lasting effects, instead of low frequency rTMS. Given that rTMS of the ATL can be annoying to the subject, as it induces muscle activation, we decided to use a protocol that lasted just $20 \mathrm{~s}$ and with stimulation intensity well under the threshold, to avoid possible confounds not related to the experimental variables. The cTBS protocol has been shown to result in a pronounced and prolonged significant effect that may even last more than thirty minutes (e.g., Huang et al., 2005; Tarnutzer et al., 2013; Bruckner et al., 2013). Moreover the vertex, instead of the occipital pole was used as control site as in many previous studies (for review see Sandrini et al., 2011).

Since we adopted the vertex (and not the occipital pole) as the control site it was unnecessary to predict a dissociation between the impairment of all kinds of visual stimuli after cTBS of the primary visual area and selective impairment of written words and pictures after cTBS of the right and left ATLs; indeed we could simply compare the effects of the cTBS applied over the right and left ATLs on the semantic processing of words and pictures representing the same concepts. We deemed that the use of a control site made it unnecessary to use a baseline measurement, also because in a cognitive task the comparison between results obtained at the baseline and after TMS is influenced by practice effects. To rule out the influence of this confounding factor, in the rTMS studies conducted by Pobric et al. (2007, 2009, 2010a, 2010b), and by Lambon-Ralph et al. (2009), half of the participants produced their "baseline", before rTMS was applied, and the other half after the end of rTMS. This procedure, however, can correct for the influence of practice effects at the group level, but not at the individual level; therefore, we did not use it in our experiment. We predicted that, if the same abstract and amodal representations are equally subsumed by both the left and the right ATLs, then the efficiency of semantic processing for words and pictures should be equally modulated by cTBS applied to these cortical areas, but not to the vertex. If, on the other hand, the left ATL mainly subsumes verbal representations and the right ATL non-verbal representations, then an interaction between side of cTBS application and the verbal or non-verbal nature of the material to be processed should be found. In particular, the semantic processing of words should be preferentially modulated by cTBS applied over the left ATL and the efficiency of semantic processing of pictures should be mainly altered by cTBS applied over the right ATL.

\section{Material and methods}

\subsection{Participants}

Eighteen right-handed participants (10 females and 8 males; mean age $=24.9$ years, $S D=2.5$ ) took part in the experiment. The presence of major systemic and neurological illnesses was excluded in all of them. All had normal or corrected-to-normal vision. All subjects gave their informed consent to participate in the study. The experimental procedures were approved by the Ethics Committee of Santa Lucia Foundation (Rome, Italy).

\subsection{Stimuli}

Ninety line drawings were chosen by combining the PPT (Howard and Patterson, 1992) and an abridged version of the Camel and Cactus Test (CCT) (Bozeat et al., 2000) stimuli. As Pobric et al. (2010a) we modified the CCT reducing the number of choice items to two (instead of four same category items). Both picture and word versions of the tests were used. The selected pictures and words were divided in three sets of thirty stimuli and were presented outlined in black. Furthermore, in order to avoid that perceptual, rather than properly semantic factors influenced results obtained on the pictorial task, we preferentially included items related by contextual or thematic associations and excluded items belonging to the same class. In every experimental condition the stimuli presented in words were different from the stimuli presented in pictures. The set of stimuli (words or pictures) and the site of stimulation (rATL; 1ATL, vertex) were assigned to subjects randomly.

\subsection{TMS}

A Magstim Super Rapid magnetic stimulator (Magstim Company) connected with a figure-of-eight coil with a $70-\mathrm{mm}$ diameter was used to deliver cTBS (Huang et al., 2005). Three-pulse bursts at $50 \mathrm{~Hz}$ repeated every $200 \mathrm{~ms}$ were delivered at $80 \%$ of the active motor threshold (AMT) for $20 \mathrm{~s}$ over right ATL, left ATL or vertex (300 pulses) as reported in Fig. 1B. The effect of cTBS with these stimulation frequency, intensity and duration last more than 15 min (e.g., Huang et al., 2005). It should be reported that, while all previous experiments that used cTBS found significant inhibitory effects using as stimulation intensity $80 \%$ (e.g., Huang et al., 2005; Nyffeler et al., 2006; Franca et al., 2006; Van Nuenen et al., 2012; Tarnutzer et al., 2013) or 70\% (Goldsworthy et al., 2013) of AMT, Bruckner et al. (2013) found that reaction times were not influenced by cTBS applied with $80 \%$ MT, but slowed only with $90 \%$. Accepted recommendations for the use and safety of non-invasive brain stimulation were applied (Rossi et al., 2009). AMT was tested over the motor cortex of the corresponding hemisphere for right and left ATL sites, respectively. Electromyographic traces were recorded from the first dorsal interosseous muscle of the contralateral hand using 9-mm diameter $\mathrm{Ag}-\mathrm{AgCl}$ surface cup electrodes. The active electrode was placed over the muscle belly and the reference electrode over the metacarpophalangeal joint of the index finger. Responses were amplified with a Digitimer D360 amplifier (Digitimer Ltd) through filters set at $20 \mathrm{~Hz}$ and $2 \mathrm{kHz}$ with a sampling rate of $5 \mathrm{kHz}$ and then were recorded by a computer using SIGNAL software (Cambridge Electronic Devices). We adopted a neuronavigation system (SofTaxic; EMS) to position the coil precisely over the stimulation sites, using individual anatomical magnetic resonance images (MRI) at 3.0 T (Siemens Magnetom Allegra; Siemens medical solution) as illustrate in Fig. 1A. Subjects were administered the semantic task in three different experimental conditions after cTBS (i.e., off-line protocol): over the left ATL (lATL) (MNI coordinates: $x=-53$; $y=+4 z=-32$ ); or over the right ATL (rATL) (MNI coordinates: $x=+53 ; y=+4 z=+32$ ) (Lambon-Ralph et al. 2009); or over the vertex (corresponding to the $\mathrm{Cz}$ position of the international electroencephalography 10-20 system) used as control site. Using a site that is not involved in the task as control condition we made sure to reproduce, in the control condition, all unspecific effects that can be induced by the cTBS protocol used in this experimental design. The coil was securely held against the left/right temple, centred over the site to be stimulated. We manipulated coil orientation (a major factor in the nature of the contraction of facial/ neck muscles) to find an orientation that minimized the discomfort to a subjective equivalent to that of the stimulation over other sites. After a training phase, in which subjects were instructed about the cTBS protocol and trained to perform the experimental procedure, they were administered cTBS over the target site and then performed the experimental task. The experimental procedure took less than $15 \mathrm{~min}$ to be performed. The cTBS conditions were performed in three different sessions, with an intersession interval of at least three days. The order of the conditions was randomized across subjects. The procedure was well tolerated by all subjects. 


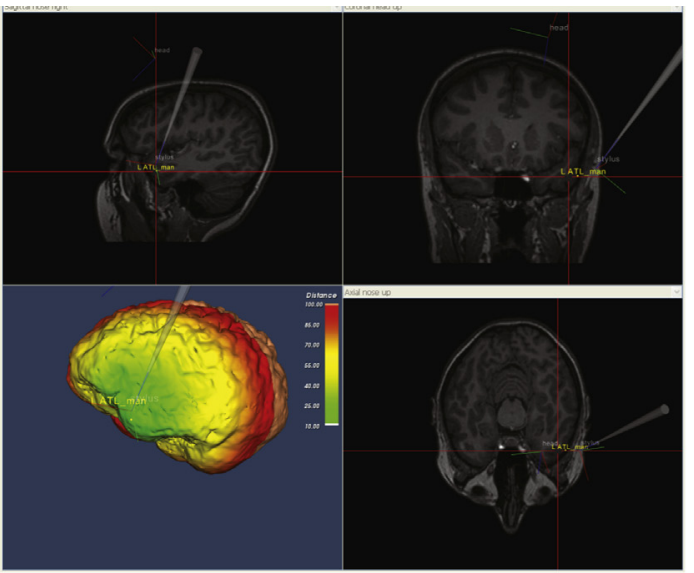

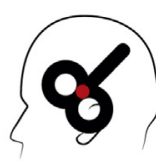

IATL

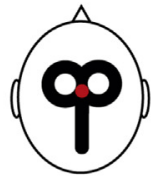

VERTEX

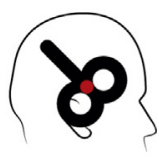

rATL

cTBS

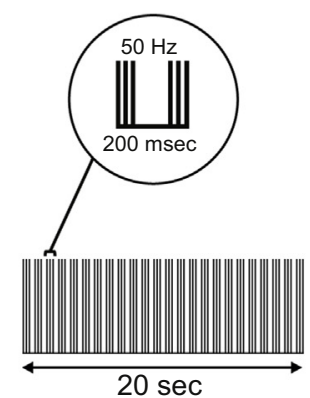

C

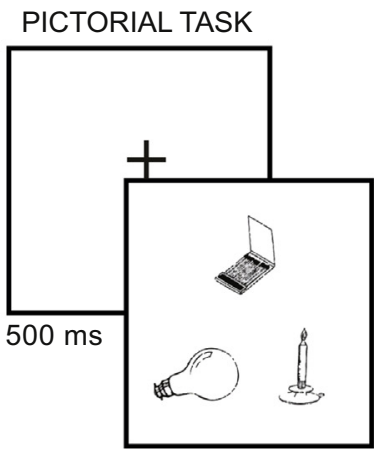

RT

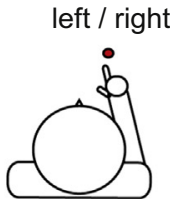

VERBAL TASK

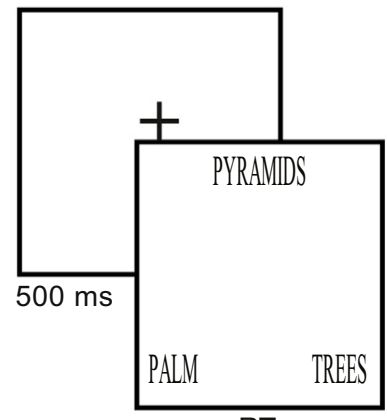

RT

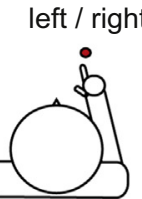

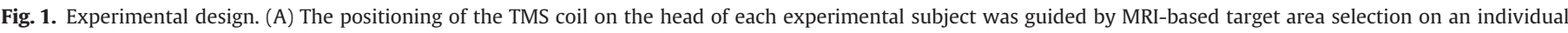

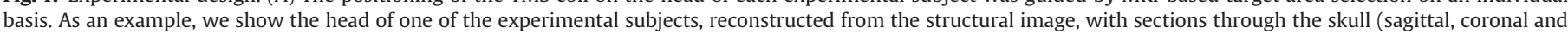

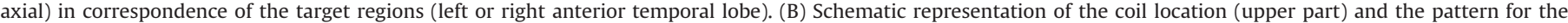
continuous theta burst stimulation (lower part). (C) Schematic representation of two trials of the semantic association task.

\subsection{Semantic association task}

Participants were tested in a quiet room. They sat comfortably on an armchair at a distance of about $80 \mathrm{~cm}$ from a computer screen; the center was aligned with the subject's eyes. In the training phase 10 pictures, not used in the experimental phase were presented to instruct the subjects on the task. During the experimental phase, participants were shown a picture or a written word, with two choice pictures or words below. Stimuli (pictures, or words) were presented until response, followed by a $500 \mathrm{~ms}$ period in which a fixation point was shown on the screen. The sequence of the stimuli was randomly assigned for each subject. The task was simply to indicate which of the two choice stimuli was more closely related to the target item, shown at the top of the screen (Fig. 1C). Subjects were asked to press as quickly as possible one of two response buttons of the keyboard that corresponded to the location of their selected item (left stimulus - left button or right stimulus - right button). In a single experimental session, participants saw sets of 30 pictures triplets or
30 words triplets. Order sites stimulation and order list were counterbalanced across experimental condition. RTs and accuracy (percentage of correct response) measurements were recorded using the Psyscope software on a PC (Fig. 1).

\section{Results}

\subsection{Results obtained in the whole experimental sample}

Subjects' accuracy and RTs on the semantic association task, were evaluated by means of two-way ANOVAs for repeated measures with Task (words semantic association vs. picture semantic association) and Stimulation Site (IATL vs. rATL vs. vertex) as within factors. When a statistically significant effect was observed, Bonferroni's tests were used for post-hoc analyses. The threshold of significance was set at $p<0.05$.

There were not significant main effects of Task $[F(1,17)=0.76$, $p=0.39]$ or Stimulation Site $[F(2,34)=0.67, p=0.5]$ and there was 


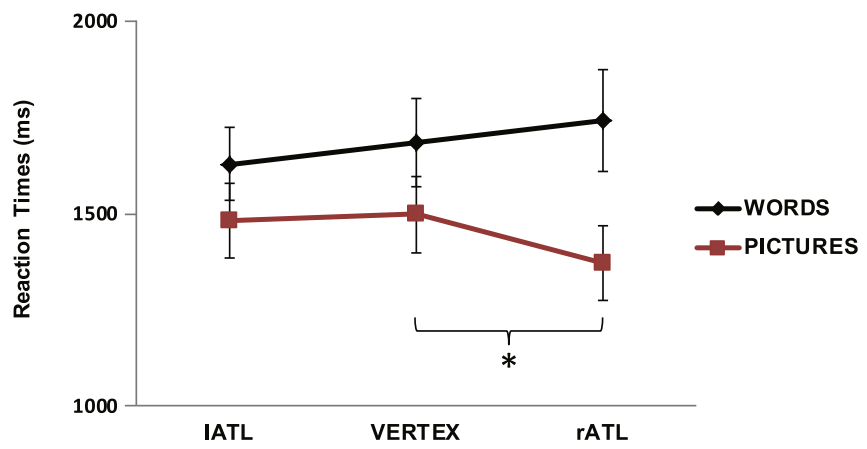

Fig. 2. Behavioural results: The graph shows the reaction times for picture and word after continuous theta burst stimulation delivered over left anterior tempora lobe (lATL), vertex, or right anterior temporal lobe (rATL). Error bars indicate the mean error standards. ${ }^{*} p<0.05$.

no significant interaction $[F(2,34)=0.58, p=0.56]$ on the accuracy. Accuracy score (\%) were: IATL $(86.7 \pm 8.7)$, vertex $(87.4 \pm 5.3)$, rATL $(87.2 \pm 5.7)$ for the word task; 1 ATL $(87.9 \pm 7.2)$, vertex $(85.7 \pm 5.9)$, rATL $(87.9 \pm 5.5)$ for the pictorial task.

There was a significant Task effect $[F(1,17)=7.7587, p=0.01]$ and a significant interaction Task $\mathrm{x}$ Stimulation Site $[F(2,34)=9.18$, $p=0.0006$ ] on RTs but we did not observe a significant Stimulation Site effect $[F(2,34)=1.06, p=0.35]$. Post hoc comparison revealed a decrease of RT after stimulation of the rATL when compared to stimulation of vertex specifically during semantic processing of pictorial material $(p<0.05)$. Fig. 2 shows the effects of cTBS delivered over IATL, rATL, or vertex on RTs.

\subsection{Results obtained on RTs by individual participants}

To evaluate how consistent the patterns obtained with word and pictures were in individual participants in the verbal and pictorial task, we calculated the algebraic differences between RTs obtained in the verbal and pictorial task after stimulation respectively of the IATL and rATL vs. vertex for each subject. Data reported in Fig. 3A show that the individual RTs for words after IATL stimulation compared to Vertex stimulation were heterogeneous. The number of subjects who presented RTs for words respectively faster or slower after $\mathrm{rATL}$ than vertex stimulation was the same $(N=9 / 18)$. On the contrary, the data reported in Fig. 3B show that the individual RTs for pictures after stimulation of the rATL follow a consistent and homogenous pattern. In 14 out of 18 subjects RTs for pictures were faster after rATL than vertex stimulation, whereas only one subject showed RTs for picture clearly longer after rATL than vertex stimulation.

\section{Discussion}

The aim of the present research was to try to clarify some discrepancies between: (a) results obtained by clinical and experimental investigations in patients in the early stages of SD and by some functional neuroimaging studies that assessed the neural correlates of verbal and nonverbal semantic processing and (b) results of rTMS experiments conducted on the right and left ATLs in normal subjects. The former results suggested that conceptual representations might be stored in a different (verbal and non-verbal) format in the left and right ATL, whereas the latter supported the hypothesis that the same amodal representations might be equally subsumed by both left and right ATLs. To clarify this issue, we conducted a TMS experiment similar to that performed by Pobric et al. (2010a) to directly investigate the role of right and left ATLs, by comparing semantic processing for the same concepts, presented in written words or pictures. However, we used a different TMS protocol i.e., cTBS and as control site we stimulated the vertex, instead of the occipital pole. Our predictions, were the following: (a) if the same amodal representations are subsumed equally by both the left and the right ATLs and the 'virtual lesion' account of the effect of cTBS stimulation is correct, than the efficiency of semantic processing for words and pictures should be reduced equally by cTBS applied to these cortical areas, but not to the vertex; (b) if, on the contrary, conceptual representations are stored in a verbal format in the left ATL and in a sensori-motor (pictorial) format in the right ATL, we should observe a greater impairment in the processing of words after a 'virtual lesion' of the left ATL and in the processing of pictures after a 'virtual lesion' of the right ATL. Our results were at variance with both predictions. But, in partial agreement with the second one, a
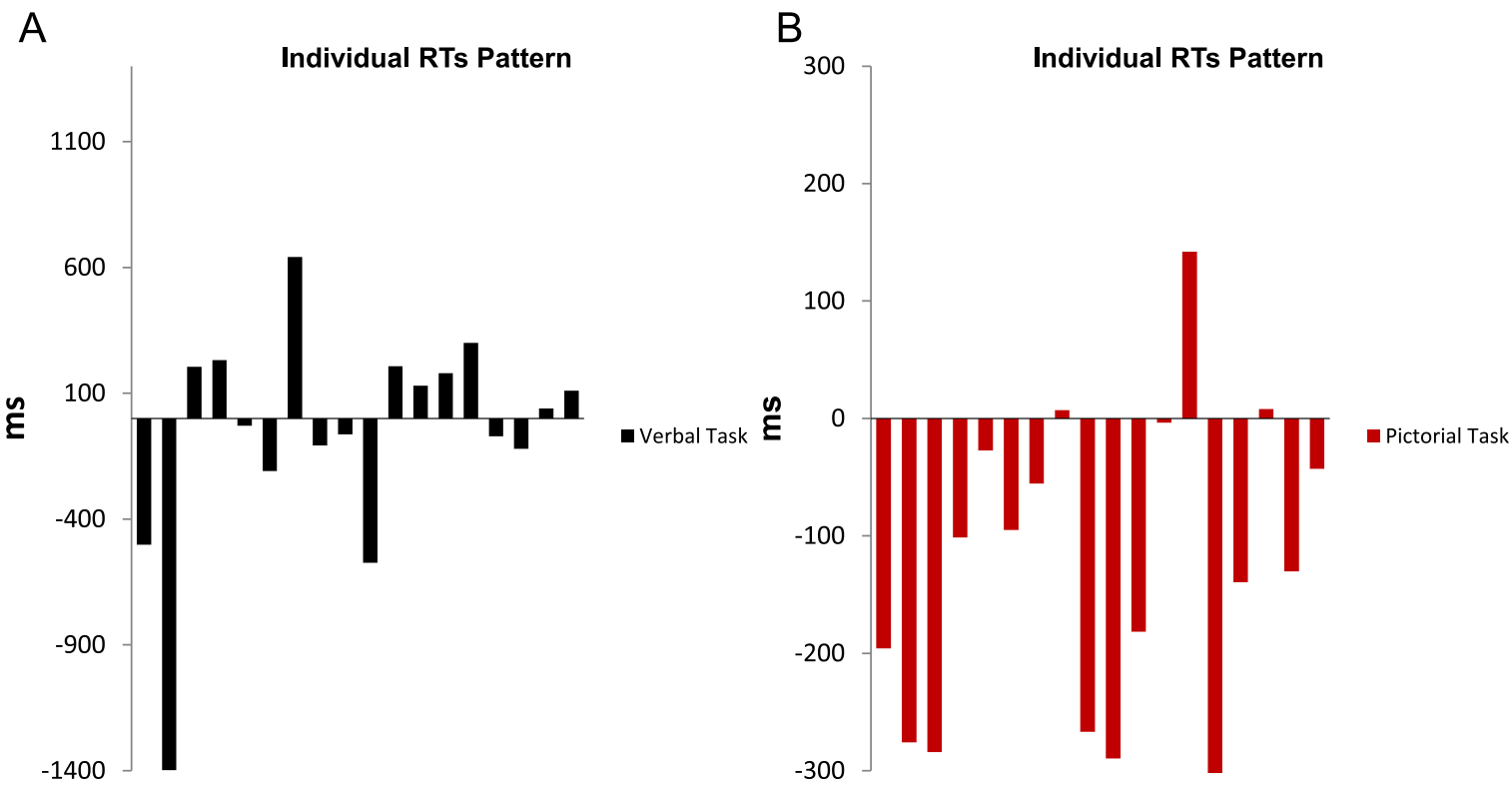

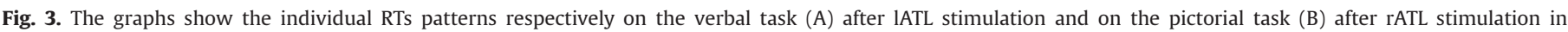

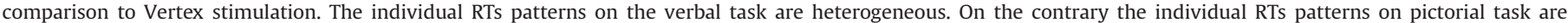
homogeneous: 14/18 subjects present RTs for pictures shorter after rATL stimulation. 
significant interaction was found between side of cTBS application and type of stimulus. This interaction suggests that different representations are stored in the right and left ATLs and that only some of these representations are influenced by cTBS application. However, contrary to our predictions and to the construct of the 'virtual lesion' used by Pobric et al. (2010a), we observed significantly faster (rather than slower) responses to pictures after application of CTBS to the right ATL, in comparison with the vertex and no difference between responses to written words after application of CTBS to the left ATL in comparison with the vertex. Both of these results were confirmed by an analysis of individual subjects RTs to pictures and written words. We found faster responses to pictures after cTBS application to the right ATL, with respect to the vertex in 14 out of 18 participants, whereas only in one subject RTs for pictures were clearly longer after rATL than vertex stimulation. On the other hand, RTs to written words were not influenced by the site (left ATL or vertex) of the cTBS application, because 9 subjects showed faster responses and 9 slower responses to words after cTBS application to the left ATL, with respect to the vertex. These unexpected results were, therefore, obtained both at the group level and at the level of individual subjects. It should be acknowledged that, since the scalp sensations at ATL are more intense than they are at vertex, the control site alone did not amend all the possible consequences of the inability to assess non-specific facilitation following ATL stimulation. For example, global facilitation combined with some mild inhibitory TMS effect in left ATL might result in an apparently specific facilitation at right ATL. Nevertheless this is not the first time that behavioural facilitation by "physiologically inhibitory protocols" has been found in language-related tasks (for a review see Vallar and Bolognini (2011)). These unexpected results can be explained in a more complex theoretical framework that goes beyond the 'virtual lesion' account and dissociates the language of physiological effects from those of behavioural effects. The use of an inhibitory TMS protocol as a virtual lesion tool has never produced a clear deficit in subject's performance, like that found in patients. In high-level functions, the inhibitory TMS protocol does not necessarily cause a disruption nor does the excitatory TMS protocol facilitate performance. Types of effects are often related to modified timing in task execution and, if a subject's performance declines, it can be explained by the level of complexity of the processing needed to solve the task. The results should be explained by considering the state of this area (Siebner et al., 2009; Silvanto and Pascual-Leone, 2008) after stimulation, and the relation between signal and noise (Miniussi et al., 2013). Repetitive stimulation is also characterized by a train of pulses whose length is dictated by frequency; therefore, such stimulation might influence neural synchronization in the cortical area involved in the execution of the task (Pasley et al., 2009; Thut and Miniussi, 2009). It can also be presumed that TMS modifies (by means of interconnections), the inhibition of the stimulated site on another site belonging to the cortical network of the investigated cognitive function, resulting in behavioural improvement. This interpretation is consistent with Pobric et al. (2010a) observation that an rTMS application to the occipital pole produced faster responses to all sorts of visual stimuli.

These findings suggest that TMS-induced effects are sensitive to subtle changes in the cortical state of the stimulated area (Siebner et al., 2009) and to the level of task difficulty (Miniussi et al., 2013). Indeed, changes in hyperpolarization alter the sensitivity of the entire system, thus including its response threshold, but these changes are ultimately expressed in subject's performance. cTBS of ATL induce a reduction in cortical excitability, which is critical for the final effects on task execution and can be translated as a reduced firing of all stimulated neurons. Nevertheless during task execution neurons that are not specific to the task execution (i.e. those that are not task related, and that produce noise during task execution) will be more affected because they are less likely to be activated by the task demands. In short, cells that fire less are more affected by cTBS, and therefore their interference with task execution is reduced. This creates a contrast in the neural signal that results in an increased signal-to-noise ratio, leading to faster responses.

In our ANOVA we found a significant task effect on RTs with shorter RTs to pictures and longer RTs to written words. Even if the mechanisms involved in reading words are still controversial and range from 'word shape' to 'serial letter recognition' or 'parallel letter recognition' (see Larson (2004) for discussion), all authors agree that that shorter words are recognized faster than longer words. Thus, the presence of rather long words could explain why picture recognition was faster than word recognition in our experiment. Two interpretations, related to this difference, can be advanced to explain the dissociation between the effects of cTBS on pictorial and verbal representations. The first interpretation is that, if the critical process which is affected by TMS occurs very early after stimulus presentation, only the faster (pictorial) task will reveal an effect, because the TMS protocol covers all of the important processing stages, but fails to cover all processes in the slower (written words) task. This interpretation is rather unlikely, given that the effect of cTBS is the same over the two ATLs and that this effect is an offline and not an online effect (that might have a different duration within trials). It is, therefore, impossible that the TMS protocol covers all of the important processing stages for pictures, but not for the (slower) written words task. The second interpretation is that a direct pictorial representation of the external world is more sensitive to these subtle modifications induced by cTBS than an indirect verbal and arbitrary representation of the same reality. This could be particularly true for context-related pictorial stimuli, such as those preferentially included in our research, in which stimulus and target are immediately visually related. Admittedly this interpretation is purely speculative and is advanced only to explain the dissociation observed in our study between the positive modifications induced in the processing of pictures by the application of cTBS over the right ATL and the lack of modifications in the processing of written words induced by application of CTBS over the left ATL.

Perhaps, the use of a visual control task would have confirmed whether the facilitation was specifically $*$ semantic* in nature. But when the experiment was planned we did not suspect that results of the theta burst TMS would affect only pictorial representations. We were, therefore, worried that the (verbal or visual) nature of a possible control task would influence the outcome of the TMS experiment. Furthermore, in order to avoid that perceptual, rather than properly semantic factors influenced results obtained on the pictorial task, we had preferentially included in our study items related by contextual or thematic associations, trying to exclude items belonging to the same class and this reinforces the hypothesis that the facilitation was specifically $*$ semantic* in nature.

Although our results are complex and partly inconsistent, they provide partial support for the hypothesis that conceptual representations are stored in a different (verbal and non-verbal) format in the left and right ATLs. Future research with on-line noninvasive brain stimulation tools, such as transcranial direct current stimulation, may allow for a more thorough investigation of the role of ATLs in ongoing cognitive semantic process.

\section{References}

Antonucci, S. M., Beeson, P. M., Labiner, D. M., \& Rapcsak, S. Z. (2008). Lexical retrieval and semantic knowledge in patients with left inferior temporal lobe lesions. Aphasiology, 22, 281-304. 
Bozeat, S., Lambon Ralph, M. A., Patterson, K., Garrard, P., \& Hodges, J. R. (2000). Non-verbal semantic impairment in semantic dementia. Neuropsychologia, 38 1207-1215.

Brückner, S., Kiefer, M., \& Kammer, T. (2013). Comparing the after-effects of continuous theta burst stimulation and conventional $1 \mathrm{~Hz}$ rTMS on semantic processing. Neuroscience, 13(233), 64-71.

Damasio, A. R. (1989). Time-locked multiregional retroactivation: a systems level proposal for the neural substrates of recall and recognition. Cognition, 33, $25-62$

Damasio, A. R. (1990). Category-related recognition defects as a clue to the neural substrates of knowledge. Trends Neurosci., 13, 95-98.

Franca, M., Koch, G., Mochizuki, H., Huang, Y. Z., \& Rothwell, J. C. (2006). Effects of theta burst stimulation protocols on phosphene threshold. Clin. Neurophysiol., 117(8), 1808-1813.

Fuggetta, G., Rizzo, S., Pobric, G., Lavidor, M., \& Walsh, V. J. (2009). Functional representation of living and nonliving domains across the cerebral hemispheres: a combined event-related potential/transcranial magnetic stimulation study. Cogn. Neurosci., 21(2), 403-414.

Gainotti, G. (2011). The organization and dissolution of semantic-conceptual knowledge: Is the 'amodal hub' the only plausible model? Brain Cogn., 75 , 299-309.

Gainotti, G. (2012). The format of conceptual representations disrupted in semantic dementia: a position paper. Cortex, 48, 521-529.

Goldsworthy, M. R., Pitcher, J. B., \& Ridding, M. C. (2013). Neuroplastic modulation of inhibitory motor cortical networks by spaced theta burst stimulation protocols. Brain Stimul., 6(3), 340-345.

Gorno-Tempini, M. L., Rankin, K. P., Woolley, J. D., Rosen, H. J., Phengrasamy, L., \& Miller, B. L. (2004). Cognitive and behavioral profile in a case of right anterior temporal lobe neurodegeneration. Cortex, 40, 631-644.

Graham, K. S., Patterson, K., \& Hodges, J. R. (1995). Progressive pure anomia: insufficient activation of phonology by meaning. Neurocase, 1, 25-38.

Hodges, J. R., Patterson, K., Oxbury, S., \& Funnell, E. (1992). Semantic dementia: progressive fluent aphasia with temporal lobe atrophy. Brain, 115, 1783-1806.

Howard, D., \& Patterson, K. (1992). Pyramis and Palm Trees: Access from Pictures and Words. Bury St Edmunds, UK. Thames Valley Test Company.

Huang, Y. Z., Edwards, M. J., Rounis, E., Bhatia, K. P., \& Rothwell, J. C. (2005). Theta burst stimulation of the human motor cortex. Neuron, 2005(45), 201-206.

Knecht, S., Flöel, A., Dräger, B., Breitenstein, C., Sommer, J., Henningsen, H., Ringelstein, E. B., \& Pascual-Leone, A. (2002). Degree of language lateralization determines susceptibility to unilateral brain lesions. Nat. Neurosci., 7, 695-699.

Lambon Ralph, M. A. McClelland, J. L., Patterson, K., Galton, C. J., \& Hodges, J. R. (2001). No right to speak? The relationship between object naming and semantic impairment: neuropsychological evidence and a computational model. J. Cogn. Neurosci., 13, 341-356.

Lambon Ralph, M. A., \& Patterson, K. (2008). Generalization and differentiation in semantic memory: insights from semantic dementia. Ann. N. Y. Acad. Sci., 1124, 61-76.

Lambon Ralph, M. A., Pobric, G., \& Jefferies, E. (2009). Conceptual knowledge is underpinned by the temporal pole bilaterally: convergent evidence from rTMS Cereb. Cortex, 19, 832-838.

Larson, K. (2004). The Science of Word Recognition or how I Learned To Stop Worryng and Love The Bouma Advanced Reading Technology. Microsoft Corporation.

Mesulam, M. M., Wieneke, C., Hurley, R., Rademaker, A., Thompson, C., Weintraub S., \& Rogalski, E. (2013). Words and objects at the tip of the left temporal lobe in primary progressive aphasia. Brain, 136, 601-618.
Miniussi, C., Harris, J. A., \& Ruzzoli, M. (2013). Modelling non-invasive brain stimulation in cognitive neuroscience. Neurosci. Biobehav. Rev. 37, 1702-1712.

Miniussi, C., Ruzzoli, M., \& Walsh, V. (2010). The mechanism of transcranial magnetic stimulation in cognition. Cortex, 46, 128-130.

Nyffeler, T., Wurtz, P., Lüscher, H. R., Hess, C. W., Senn, W., Pflugshaupt, T., von Wartburg, R., Lüthi, M., \& Müri, R. M. (2006). Extending lifetime of plastic changes in the human brain. Eur. J. Neurosci., 24(10), 2961-2966.

Pasley, B. N., Allen, E. A., \& Freeman, R. D. (2009). State-dependent variability of neuronal responses to transcranial magnetic stimulation of the visual cortex. Neuron, 62, 291-303.

Patterson, K., Nestor, P. J., \& Rogers, T. T. (2007). Where do you know what you know? The representation of semantic knowledge in the human brain. Nat. Rev. Neurosci., 8, 976-987.

Pobric, G., Jefferies, E., \& Lambon Ralph, M. A. (2007). Anterior temporal lobes mediate semantic representation: mimicking semantic dementia by using rTMS in normal participants. Proc. Natl. Acad. Sci. USA, 104, 20137-20141.

Pobric, G., Jefferies, E., \& Lambon Ralph, M. A. (2010a). Amodal semantic representations depend on both anterior temporal lobes: evidence from repetitive transcranial magnetic stimulation. Neuropsychologia, 48, 1336-1342.

Pobric, G., Jefferies, E., \& Lambon Ralph, M. A. (2010b). Category-specific versus category-general semantic impairment induced by transcranial magnetic stimulation. Curr. Biol., 20(10), 964-968.

Pobric, G., Lambon Ralph, M. A., \& Jefferies, E. (2009). The role of the anterior temporal lobes in the comprehension of concrete and abstract words: rTMS evidence. Cortex, 45, 1104-1110.

Rossi, S., Hallett, M., Rossini, P. M., \& Pascual-Leone, A. (2009). Safety of TMS Consensus Group. (2009). Safety, ethical considerations, and application guidelines for the use of transcranial magnetic stimulation in clinical practice and research. Clin. Neurophysiol., 120, 2008-2039.

Sandrini, M., Umiltà, C., \& Rusconi, E. (2011). The use of transcranial magnetic stimulation in cognitive neuroscience: a new synthesis of methodological issues. Neurosci. Biobehav. Rev., 35, 516-536.

Siebner, H. R., Hartwigsen, G., Kassuba, T., \& Rothwell, J. C. (2009). How does transcranial magnetic stimulation modify neuronal activity in the brain? Implications for studies of cognition. Cortex, 45, 1035-1042.

Silvanto, J., \& Pascual-Leone, A. (2008). State-dependency of transcranial magnetic stimulation. Brain Topogr., 21, 1-10.

Snowden, J. S., Thompson, J. C., \& Neary, D. (2004). Knowledge of famous faces and names in semantic dementia. Brain, 127, 860-872.

Snowden, J. S., Thompson, J. C., \& Neary, D. (2012). Famous people knowledge and the right and left temporal lobes. Behav. Neurol., 25, 35-44.

Thut, G., \& Miniussi, C. (2009). New insights into rhythmic brain activity from TMSEEG studies. Trends Cogn. Sci., 13, 182-189.

Tarnutzer, A. A., Lasker, A. G., \& Zee, D. S. (2013). Continuous theta-burst stimulation of the right superior temporal gyrus impairs self-motion perception. Exp. Brain Res., 230(3), 359-370.

Vallar, G., \& Bolognini, N. (2011). Behavioural facilitation following brain stimulation: implications for neurorehabilitation. Neuropsychol. Rehab., 21, 618-649.

Van Nuenen, B. F., Kuhtz-Buschbeck, J., Schulz, C., Bloem, B. R., \& Siebner, H. R (2012). Weight-specific anticipatory coding of grip force in human dorsal premotor cortex. J. Neurosci., 32(15), 5272-5283. 